\title{
SIMPLY CONNECTED SETS $\uparrow$
}

BY

R. E. BASYE

The class of simply connected sets, which is the object of study of the present paper, is closely related to the class of unicoherent sets introduced by Vietoris $\ddagger$ and Kuratowski.§ A connected set is unicoherent if, however it be expressed as the sum of two connected and relatively closed subsets, the common part of the latter is connected. For locally connected metric sets the two classes coincide. In order that a connected and locally arcwise connected subset $M$ of the plane be simply connected, it is necessary and sufficient that the interior of every simple closed curve lying in $M$ be a subset of $M$. The notion of simple connectedness in the weak sense is also defined. The properties of sets of these types have a variety of applications and furnish an interesting background for a number of well known theorems.

I wish to express my thanks to Professor R. L. Moore, who greatly encouraged me in the writing of this paper.

A set $A$ is closed in a set $B$ if $A$ lies in $B$ and contains every point of $B$ which is a limit point of $A$. If $A$ is closed in $B$ it is called a relatively closed subset of $B$. If $F$ and $G$ are subsets of a reference space $S, F$ is said to be a closed subset of $G$ if $F$ lies in $G$ and is closed in $S$.

In a space $S$ let $C$ be a connected set and $L$ any set whatever. $L$ is said to disconnect, or separate $C$ if $C-C \cdot L$ is not connected. Let $H$ and $K$ be two mutually exclusive subsets of $C$ neither of which intersects $L$. Then $L$ is said (1) to separate $H$ from $K$ in $C$ if $C-C \cdot L$ can be expressed as the sum of two mutually separated sets which contain $H$ and $K$ respectively, (2) to disconnect $H$ from $K$ in $C$ if every connected subset of $C$ that intersects both $H$ and $K$ contains a point of $L$, and (3) to weakly disconnect $H$ from $K$ in $C$ if every connected and relatively closed subset of $C$ that intersects both $H$ and $K$ contains a point of $L$.

The three types of separation defined in (1), (2), and (3) are successively weaker. However, if $C$ is a locally connected metric space, and $L \cdot C$ is closed in $C$, the three types are completely equivalent.

A connected set $M$ is said to be simply connected if for each pair of points $A$ and $B$ of $M$, and any relatively closed subset $L$ of $M$ that separates $A$ from

$\dagger$ Presented to the Society, October 28, 1933; received by the editors January 19, 1935.

$\ddagger$ Ueber stetige Abbildungen einer Kugelfäche, Akademie van Wetenschappen, Amsterdam, Proceedings, vol. 29 (1926), p. 445. Vietoris uses the term "henkellos."

$\S$ Une caractêrisation topologique de la surface de la sphère, Fundamenta Mathematicae, vol. 13 (1929), p. 308. 
$B$ in $M$, there exists a connected subset of $L$ which separates $A$ from $B$ in $M$. This definition becomes the criterion for a connected set to be simply connected in the weak sense if "separates" is replaced throughout by "weakly disconnects." The properties of being simply connected and simply connected in the weak sense are intrinsic and topologically invariant.

Every simply connected set is simply connected in the weak sense. If a metric and locally connected space is simply connected in the weak sense, it is simply connected.

A simply connected metric space need not be locally connected. For example, the plane set consisting of the origin and the points $(x, y)$ for which $0<x \leqq 1, y=\sin (1 / x)$, is simply connected but not locally connected. If in addition, however, the space is locally compact, its local connectivity follows.

In the definition of simply connected sets the separating set $L$ was required to be closed. It can be shown that, for metric sets, this requirement may be omitted. A similar remark does not apply to the definition of sets that are simply connected in the weak sense.

THEOREM 1. If $A$ and $B$ are two points of a simply connected metric space $M$, and $G=\left\{g_{i}\right\}$ is a countable collection of mutually exclusive closed sets no one of which separates $A$ from $B$ in $M$, and $G^{*}$ is compact in $M$, then $G^{*}$ does not separate $A$ from $B$ in $M$.

For suppose $G^{*}$ does separate $A$ from $B$ in $M$. Then there exists a closed subset $L$ of $G^{*}$ which separates $A$ from $B$ in $M$. Since $M$ is simply connected, there exists a component $\lambda$ of $L$ which separates $A$ from $B$ in $M$. The continuum $\lambda$ is not a subset of any element of $G$ since no element of $G$ separates $A$ from $B$ in $M$. Thus $\lambda$ is the sum, $\sum \lambda \cdot g_{i}$, of a countable number (greater than one) of mutually exclusive closed sets. But $\lambda$ is compact. This contradicts a theorem of Sierpinski. $\dagger$

THEOREM 2. If $A$ and $B$ are two points of a locally connected metric space $M$, and $H$ and $K$ are two closed sets neither of which separates $A$ from $B$ in $M$, and if the complementary domain $D$ of $H \cdot K$ that contains $A+B$ is simply connected, then $H+K$ does not separate $A$ from $B$ in $M$.

THEOREM 3. Let $A$ and $B$ be two points of a connected and locally arcwise connected metric space $S$, and let $G=\left\{g_{i}\right\}$ be a countable collection of closed sets such that (1) the common part of every pair of elements of $G$ is the closed set $H$ (which may be vacuous), (2) if $b_{1}$ and $b_{2}$ are two arcs from $A$ to $B$ that lie in $S-H$, then $b_{1}+b_{2}$ lies in a compact set which is simply connected in the weak sense and whose closure contains no point of $H$, and (3) $G^{*}$ is locally compact. If no element of $G$ separates $A$ from $B$ in $S$, then $G^{*}$ does not separate $A$ from $B$ in $S$.

† Un théorème sur les continus, Tôhoku Mathematical Journal, vol. 13 (1918), pp. 300-303. 
On the contrary supposition there exists a subset $F$ of $G^{*}$ which is closed, contains $H$, separates $A$ from $B$ in $S$, and is irreducible with respect to these three properties. Denote by $G^{\prime}$ the collection $\left\{g_{i}^{\prime}\right\}$, where $g_{i}^{\prime}=F \cdot g_{i}$. The set $F-H$, which is locally self-compact, is the sum of the countable number of relatively closed subsets $g_{i}^{\prime}-H$. Hence, by a theorem of R. L. Moore, $\dagger$ there exists a set $g_{k}{ }^{\prime}-H$ that contains a point which is not a limit point of $(F-H)-\left(g_{k}^{\prime}-H\right)$. It easily follows that there exists an arc $b_{1}$ from $A$ to $B$ which has no point in common with any element of $G^{\prime}$ except $g_{k}^{\prime}$. Moreover, since by hypothesis $g_{k}$ does not separate $A$ from $B$ in $S$, there exists an arc $b_{2}$ from $A$ to $B$ which has no point in common with $g_{k}{ }^{\prime}$. The continuum $b_{1}+b_{2}$ lies in a compact set $W$ which is simply connected in the weak sense and such that $\bar{W} \cdot H=0$. The set $W \cdot F$ separates $A$ from $B$ in $W$. Hence some component $w$ of $W \cdot F$ weakly disconnects $A$ from $B$ in $W$, and thus intersects both $b_{1}$ and $b_{2}$. Consequently $w$ contains points of at least two elements of $G^{\prime}$. Thus the compact continuum $\bar{w}$ is the sum, $\sum \bar{w} \cdot g_{i}^{\prime}$, of a countable number (more than one) of mutually exclusive closed sets, contrary to a theorem of Sierpiński.ł

THEOREM 4. If a metric space is simply connected in the weak sense, it is unicoherent.

That the converse of Theorem 4 is not true can be illustrated by simple examples of compact plane continua.

THEOREM 5. A necessary and sufficient condition that a connected and locally connected metric space $M$ be simply connected is that, if $K$ is any subcontinuum of $M$, the boundary of every complementary domain of $K$ be connected.

The condition is necessary. For let $M$ be simply connected and suppose there exists a continuum $K$ such that the boundary $B$ of a complementary domain $D$ of $K$ is not connected. Since $M$ is connected and locally connected, the boundary of every complementary domain of $K$ is a non-vacuous subset of $K$. Hence $M-D$ is a continuum. Thus $M$ is the sum of two continua, $M-D$ and $\bar{D}$, whose intersection is the disconnected set $B$. It follows from Theorem 4 that $M$ is not simply connected, contrary to hypothesis.

Assume next that the condition is satisfied. Let $L$ be a closed set which separates the point $P$ from the point $Q$ in $M$. Denote by $D$ the complementary domain of $L$ that contains $P$, by $B$ the boundary of $D$, and by $\Delta$ the complementary domain of the continuum $D+B$ that contains $Q$. The boundary $\beta$ of $\Delta$ is a subset of $B$. But $B$ is a subset of $L$. And by assumption $\beta$ is con-

$\dagger$ Foundations of Point Set Theory, American Mathematical Society Colloquium Publications, vol. 13, p. 11, Theorem 15 .

$\ddagger$ Loc. cit. 
nected. Thus $\beta$ is a connected subset of $L$ that separates $P$ from $Q$ in $M$. Therefore $M$ is simply connected.

It has been proved by $\mathrm{C}$. Kuratowski† that for a connected and locally connected metric space $M$ the following three propositions are equivalent:

A. If $K$ is any subcontinuum of $M$, the boundary of every complementary domain of $K$ is connected.

B. However $M$ be expressed as the sum of two continua, the intersection of the latter is connected.

C. If $H$ and $K$ are any two mutually exclusive closed subsets of $M$, and $P$ and $Q$ are points of $H$ and $K$ respectively, there exists a continuum which separates $P$ from $Q$ in $M$ and contains no point of $H+K$.

Thus, in view of Theorem 5, we have the following result.

THEOREM 6. Each of the conditions $\mathrm{B}$ and $\mathrm{C}$ is necessary and sufficient that a connected and locally connected metric space be simply connected.

We next prove a result which, for the compact case, is a consequence of a closely related theorem $\ddagger$ of $\mathrm{W}$. A. Wilson.

TheOREM 7. Let $M$ be a metric, connected, and locally arcwise connected space. If every simple closed curve lying in $M$ is contained in a subset of $M$ which is simply connected in the weak sense, then $M$ is simply connected.

For suppose $M$ is not simply connected. By Theorem 5 there exists a continuum $K$ having a complementary domain $D$ whose boundary $B$ is not connected. Let $B=B_{1}+B_{2}$, where $B_{1}$ and $B_{2}$ are mutually separated sets. Let $P_{1} P_{2}$ be an arc contained in $D$ except for its end points, which lie in $B_{1}$ and $B_{2}$ respectively. Let $F$ be a closed set which separates $B_{1}$ from $B_{2}$ in $M$. Denote by $Q_{1}$ and $Q_{2}$ the first points of $F$ on $P_{1} P_{2}$ in the orders from $P_{1}$ to $P_{2}$ and from $P_{2}$ to $P_{1}$ respectively. About each point $X$ of $K$ consider a connected region $R_{X}$ such that (1) $R_{X} \cdot F=0$ if $X \ddagger F$, (2) $R_{X} \cdot \bar{D}=0$ if $X \subset F$, (3) $R_{X} \cdot\left(P_{1} P_{2}\right)=0$ if $P_{1} \neq X \neq P_{2}$, and (4) $R_{P_{1}} \cdot\left(Q_{1} P_{2}\right)=R_{P_{2}} \cdot\left(Q_{2} P_{1}\right)=0$. The sum of these regions is a connected domain $\Delta$ whose intersection with $F$ has no point in common with $\bar{D}$. Let $h$ be an arc in $\Delta$ with end points $P_{1}$ and $P_{2}$. Denote by $P_{2}^{\prime}$ the first point of $h$ in the order from $P_{1}$ to $P_{2}$ which lies on $P_{2} Q_{2}$, and by $P_{1}^{\prime}$ the first point of $h$ in the order from $P_{2}^{\prime}$ to $P_{1}$ which lies on $P_{1} Q_{1}$. Denote by $u$ the subarc $P_{1}^{\prime} P_{2}^{\prime}$ of $P_{1} P_{2}$, and by $v$ the subarc $P_{1}^{\prime} P_{2}^{\prime}$ of $h$.

By hypothesis the simple closed curve $u+v$ lies in a subset $N$ of $M$ which is simply connected in the weak sense. The set $F$ separates $P_{1}^{\prime}$ from $P_{2}^{\prime}$ in

$\dagger$ Sur les continus de Jordan et le théorème de M. Brouwer, Fundamenta Mathematicae, vol. 8 (1926), pp. 148-150.

$\ddagger$ On unicoherency about a simple closed curve, American Journal of Mathematics, vol. 55 (1933), pp. 135-145, Theorem of $\$ 11$. 
$M$ since it separates $P_{1}$ from $P_{2}$ in $M$. Hence the set $F \cdot N$, which is closed in $N$, separates $P_{1}^{\prime}$ from $P_{2}^{\prime}$ in $N$. Consequently some component $F_{0}$ of $F \cdot N$ weakly disconnects $P_{1}^{\prime}$ from $P_{2}^{\prime}$ in $N$. Thus $F_{0}$ contains a point $U$ of $u$ and a point $V$ of $v$. But $U \subset D$ and $V \nsubseteq D$. It follows that $F_{0}$ contains a point of the boundary of $D$, contrary to the construction of $F$.

Coroltary 1. A metric, connected, and locally arcwise connected space $M$ is a simple closed curve if and only if it fails to be simply connected but becomes simply connected upon the omission of any one of its points.

CoRollary 2. Let $M$ be a metric and locally arcwise connected space. If $\sigma$ is a monotonic ascending sequence of simply connected subdomains of $M$, then the sum of the domains of $\sigma$ is also simply connected.

THEOREM 8. If $D$ is a connected subdomain of a compact, metric, continuous curve $M$, and the boundary $B$ of $D$ has at least $n$ components ( $n$ an integer), then $D$ contains a compact continuum $K$ such that every subset of $M$ which separates $K$ from $B$ in $M$ has at least $n$ components.

Let $B$ be expressed as the sum of $n$ mutually separated sets $B_{1}, \cdots, B_{n}$. Let $F_{i}(i=1, \cdots, n)$ be a closed set which separates $B_{i}$ from $B-B_{i}$ in $M$. There exists a continuum $K$ which lies in $D$ and contains the closed set $\left(F_{1}+\cdots+F_{n}\right) \cdot D$. Let $P$ be a point of $K$ and consider an $\operatorname{arc} P Q_{i}(i=1$, $\cdots, n)$ that is contained in $D$ except for the point $Q_{i}$, which lies in $B_{i}$. Let $P_{i}$ be the first point of $Q_{i} P$ that lies in $K$. If $L$ is a subset of $M$ which separates $K$ from $B$ in $M$, then $L$ must contain a point $Z_{i}$ of the $\operatorname{arc} P_{i} Q_{i}(i=1, \cdots, n)$. Denote by $\lambda_{i}$ the component of $L$ that contains $Z_{i}$. These components are subsets of $D$. Moreover no two of them coincide. For if $\lambda_{i}=\lambda_{j}(i \neq j)$, then $\lambda_{i}+Q_{i} Z_{i}+Q_{j} Z_{j}$ is a connected set which intersects $B_{i}$ and $B_{j}$ and contains no point of $F_{i}$, contrary to the construction of $F_{i}$. It follows that $L$ has at least $n$ components.

THEOREM 9. A compact, metric, continuous curve $M$ is simply connected if and only if every two mutually exclusive subcontinua of $M$ can be separated in $M$ by a third subcontinuum of $M$.

Theorem 9 can be proved with the aid of Theorems 5 and 8 .

The following variation of Theorem 9 may be stated: A compact, metric, continuous curve $M$ is simply connected if and only if, given any two mutually exclusive subcontinua $H$ and $K$ of $M$, there exists but one component of $M-(H+K)$ whose closure intersects both $H$ and $K$.

THEOREM 10. A compact, metric, one-dimensional continuum $M$ is simply connected in the weak sense if and only if there exists but one irreducible subcontinuum of it between any two of its points. 
The condition is necessary. For let $M$ be simply connected in the weak sense and suppose there exist in $M$ two distinct continua, $L_{1}$ and $L_{2}$, each of which is irreducible between the point $A$ and the point $B$. Clearly $A$ and $B$ lie in different components of $I=L_{1} \cdot L_{2}$. Let $I=I_{A}+I_{B}$, where $I_{A}$ and $I_{B}$ are mutually separated sets containing $A$ and $B$ respectively. Let $F$ be a closed subset of $M$ which separates $I_{A}$ from $I_{B}$ in $M$. About each point $P$ of $F$ consider a domain (relative to $M$ ) whose closure contains no point of $I$ and such that its boundary is totally disconnected. There exists a finite number of these domains, $D_{1}, \cdots, D_{n}$, whose sum $D$ covers $F$. If $\beta_{i}$ denotes the boundary of $D_{i}$, the set $\beta_{1}+\cdots+\beta_{n}$ is totally disconnected. Hence the boundary $\beta$ of $D$, being a subset of $\beta_{1}+\cdots+\beta_{n}$, is totally disconnected. Now $F$ separates $A$ from $B$ in $M$, and $\beta$ separates $A+B$ from $F$ in $M$; hence $\beta$ separates $A$ from $B$ in $M$. Consequently there exists a component $Q$ of $\beta$ which weakly disconnects $A$ from $B$ in $M$, and $Q$ must be a point. Since $Q$ weakly disconnects $A$ from $B$ in $M$, it must intersect both $L_{1}$ and $L_{2}$. Thus $Q$ is a point of $I$, contrary to the construction of $\beta$.

The sufficiency of the condition is obvious.

THEOREM 11. In a locally compact, locally connected, simply connected, metric space $M$ let $H$ and $K$ be two closed sets of which $H$ is compact and whose intersection $T$ is totally disconnected. If $A$ and $B$ are points of $H-T$ and $K-T$ respectively, there exists a compact continuous curve, lying in $M$, which separates $A$ from $B$ in $M$ and contains no point of $(H+K)-T$.

There exists a domain $D$ which contains $H$ and is compact in $M$, and there exists a closed subset $F$ of $D$ which separates $H-T$ from $K-T$ in $M$. The set $F$ is compact. Since $M$ is simply connected, there exists a component $\phi$ of $F$ which separates $A$ from $B$ in $M$. We shall construct a compact continuous curve which contains $\phi$ and has no point in common with $(H+K)-T$.

Let $\Delta_{1}, \Delta_{2}, \cdots$ be a sequence of compact domains closing down on $\phi \cdot T$ such that $\bar{\Delta}_{i+1} \subset \Delta_{i}(i=1,2, \cdots)$. Denote by $L_{0}$ the compact closed set $\phi \cdot\left(M-\Delta_{1}\right)$, and by $L_{i}(i=1,2, \cdots)$ the compact closed set $\phi \cdot\left(\bar{\Delta}_{i}-\Delta_{i+1}\right)$. For each point $P$ of $L_{i}(i=0,1,2, \cdots)$ let $r_{P}$ be a compact and connected domain containing $P$ such that (1) $\bar{r}_{P} \cdot(H+K)=0$, and (2) $\bar{r}_{P} \subset\left(\Delta_{i-1}-\bar{\Delta}_{i+2}\right)$ if $i>1$. By the Borel-Lebesgue theorem there exists a finite number of the closed domains $\bar{r}_{P}$ whose sum $R_{i}$ covers $L_{i}$. Thus $R_{i}$ is a compact closed set which has only a finite number of components and contains no point of $H+K$. Consequently $\dagger$ each component of $R_{i}$ can be imbedded in a compact contin-

† Special cases of the theorem required here are due to H. M. Gehman, G. T. Whyburn, W. L. Ayres, and others. For references to their results and Wilder's generalization, see R. L. Wilder, On the imbedding of subsets of a metric space in Jordan continua, Fundamenta Mathematicae, vol. 19 (1932), pp. 45-64. 
uous curve, lying in $M$, which has no point in common with $H+K$, and which, if $i>1$, is a subset of $\left(\Delta_{i-1}-\bar{\Delta}_{i+2}\right)$. The sum $S_{i}$ of these continuous curves is a closed and locally connected set which contains $L_{i}$. It readily follows that $S=\sum S_{i}+\phi \cdot T$ is a compact continuum, containing $\phi$, which is locally connected at every point of $\sum S_{i}$ and hence, since $\phi \cdot T$ is totally disconnected, at every point of $S$. Therefore $S$ is a compact continuous curve which separates $A$ from $B$ in $M$ and has no point in common with $(H+K)-T$.

A sequence of sets lying in a space $S$ is said to close down on a compact closed set $K$ if $K$ is common to all the sets of the sequence and if every domain containing $K$ contains all but a finite number of the sets of the sequence. We now prove a proposition which generalizes a theorem of $\mathrm{K}$. Borsuk $\dagger$ and is closely related to a theorem of Vietoris.

THEOREM 12. In a compact metric space $E$ let $\sigma=\left\{S_{i}\right\}$ be a sequence of sets closing down on a closed set $S$. If the sets of $\sigma$ are simply connected in the weak sense, so also is $S$.

Let $A$ and $B$ be any two points of $S$, and $F$ any relatively closed subset of $S$ which weakly disconnects $A$ from $B$ in $S$. Let $\left\{D_{i}\right\}$ be a sequence of domains closing down on $F$ such that the closure of no one of them contains $A$ or $B$. For each $i$ the closed domain $\bar{D}_{i}$ weakly disconnects $A$ from $B$ in at least one of the sets of $\sigma$. For suppose $\bar{D}_{i}$ does not weakly disconnect $A$ from $B$ in any set of $\sigma$. Then for each $j$ there exists a relative subcontinuum $C_{j}$ of $S_{j}$ which contains $A+B$ and has no point in common with $\bar{D}_{i}$. Some subsequence of $\left\{C_{j}\right\}$ has a sequential limiting set $C$. The set $C$ is a subcontinuum of $S$ that contains $A+B$ and has no point in common with $D_{i}$. Hence $C$ has no point in common with $F$, contrary to the fact that $F$ weakly disconnects $A$ from $B$ in $S$.

For each $i$ let $S_{n_{i}}$ be the first set of $\sigma$ such that $\bar{D}_{i}$ weakly disconnects $A$ from $B$ in $S_{n_{i}}$. Since, by hypothesis, $S_{n_{i}}$ is simply connected in the weak sense, there exists a component $d_{i}$ of $S_{n_{i}} \cdot \bar{D}_{i}$ which weakly disconnects $A$ from $B$ in $S_{n_{i}}$. Some subsequence of $\left\{d_{i}\right\}$ has a sequential limiting set $d$. The continuum $d$ is a subset of $F$ since $\left\{D_{i}\right\}$ closes down on $F$. Furthermore $d$ weakly disconnects $A$ from $B$ in $S$. For suppose $L$ is a subcontinuum of $S$ which contains $A+B$ but no point of $d$. Then there exists an integer $r$ such that $d_{r}$ and $L$ are mutually exclusive. But this implies that $d_{r}$ cannot weakly disconnect $A$ from $B$ in any set of $\sigma$, contrary to what was shown above.

\footnotetext{
† Quelques théorèmes sur les ensembles unicohérents, Fundamenta Mathematicae, vol. 17 (1931), p. 208.

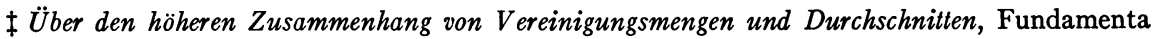
Mathematicae, vol. 19 (1932), p. 266.
} 
Thus $d$ is a connected subset of $F$ which weakly disconnects $A$ from $B$ in $S$. Therefore $S$ is simply connected in the weak sense.

COROLLARY. If, in a metric space, $\sigma$ is a monotonic descending sequence of compact continua which are simply connected in the weak sense, the product of the sets of $\sigma$ is also simply connected in the weak sense.

We state next the fundamental lemma for simply connected subsets of the plane.

Lemma K. A plane continum consisting of a simple closed curve and its interior is simply connected. $\dagger$

We note that a consequence of Lemma $\mathrm{K}$ and the Corollary of Theorem 12 is the following result due to Urysohn:

THEOREM K. Every compact plane continuum which does not separate the plane is simply connected in the weak sense. $\ddagger$

We note further that Theorems 4 and $\mathrm{K}$ imply the following theorem of S. Janiszewski: If two compact plane continua intersect in a disconnected set, their sum separates the plane.§

The following generalization of Theorem $\mathrm{K}$ can be proved by considering an inversion of the plane.

TheOREM 13. If $A$ and $B$ are two points of a compactly connected $\|$ plane continuum $M$ which does not separate the plane, $F$ is a closed subset of $M$ that weakly disconnects $A$ from $B$ in $M$, and every component of $F$ is compact, then some component of $F$ weakly disconnects $A$ from $B$ in $M$.

In accordance with Urysohn's theorem every compact plane continuum which does not separate the plane is simply connected in the weak sense. We next prove that certain continua which separate the plane have the same property.

THEOREM 14. Every compact, indecomposable, plane continuum $M$ which is the common boundary of all its complementary domains is simply connected in the weak sense.

We shall show that if $A$ and $B$ are points of $M$ there exists but one sub$24^{\prime}$.

$\dagger$ For a proof of this lemma see R. L. Moore, Foundations of Point Set Theory, p. 194, Theorem

$\ddagger$ Ueber Räume mit verschwindender erster Brouwerscher Zahl, Akademie van Wetenschappen, Amsterdam, Proceedings, vol. 31 (1928), pp. 808-810.

$\S$ Sur les coupures du plan faites par les continus, Prace Matematyczno-Fizyczne, vol. 26 (1913), pp. 11-63.

\| A connected set $M$ is compactly connected if every two of its points lie together in a compact subcontinuum of M. See R. L. Moore, Foundations of Point Set Theory, p. 465. 
continuum of $M$ which is irreducible between $A$ and $B$. Suppose $L_{1}$ and $L_{2}$ are two distinct subcontinua of $M$ each of which is irreducible between $A$ and $B$. The intersection of $L_{1}$ and $L_{2}$ cannot be connected; hence $L_{1}+L_{2}$ separates the plane. Since $M$ is indecomposable there exists a point $P$ of $M-\left(L_{1}+L_{2}\right)$. Denote by $D$ a complementary domain of $L_{1}+L_{2}$ which does not contain $P$, and by $\Delta$ a complementary domain of $M$ which lies in $D$. The boundary of $D$ lies in $L_{1}+L_{2}$ and hence cannot contain $P$. Therefore $P$ cannot lie on the boundary of $\Delta$, contrary to hypothesis.

It follows that $M$ is simply connected in the weak sense.

With the aid of this result it is easy to construct examples of compact plane continua which separate the plane, are not indecomposable, and are simply connected in the weak sense. It would be interesting to find necessary and sufficient conditions for a compact plane continuum to be simply connected in the weak sense.

TheOREM 15. If $A$ and $B$ are two points of a compactly connected plane continuum $M$ which does not separate the plane, and $G$ is a countable collection of mutually exclusive closed subsets of $M$ no one of which weakly disconnects $A$ from $B$ in $M$, and $G^{*}$ is closed and compact, then $G^{*}$ does not weakly disconnect $A$ from $B$ in $M$.

The proof, based on Theorem 13, is similar to that of Theorem 1.

THEOREM 16. If $H$ and $K$ are two plane continua one of which is compact, and $G$ denotes the collection of those complementary domains of $H+K$ each of whose boundaries contains points of $H-H \cdot K$ and points of $K-H \cdot K$, then $G^{*}+H \cdot K$ is a connected set which is not disconnected by any element of $G$.

If $H \cdot K=0$ then $G$ contains only one element.

Suppose $H \cdot K \neq 0$. Consider the case where $H$ and $K$ are both compact and suppose there exists an element $D$ of $G$ such that $\left(G^{*}+H \cdot K\right)-D=N_{1}+N_{2}$, where $N_{1}$ and $N_{2}$ are mutually separated sets. The boundary of each element of $G$ is connected and therefore contains a point of $H \cdot K$. Hence there exist points $P_{1}$ and $P_{2}$ of $H \cdot K$ which lie in $N_{1}$ and $N_{2}$ respectively. At least one of the sets $N_{1}, N_{2}$ is bounded. Hence there exists a compact closed subset $F$ of $S-D$ (where $S$ denotes the plane) which separates $N_{1}$ from $N_{2}$ in $S-D$. Now $S-D$ is a compactly connected continuum which does not separate $S$. Hence, by Theorem 13, there exists a component $F_{0}$ of $F$ which weakly disconnects $P_{1}$ from $P_{2}$ in $S-D$. Hence $F_{0}$ intersects both $H$ and $K$. The continuum $F_{0}$ contains a connected set $f_{0}$ which contains no point of $H+K$ but such that both $H$ and $K$ contain at least one limit point of $f_{0}$. Hence $f_{0}$ is a subset of an element of $G$. This is a contradiction. It follows also that $G^{*}$ $+H \cdot K$ is connected. 
The case where one of the sets $H, K$ is not compact can be reduced to the one considered by an inversion of the plane about a circle lying in $D$.

It is interesting to observe that the Janiszewski theorem mentioned in connection with Theorem $\mathrm{K}$ is also a direct corollary of Theorem 16 .

Another theorem due to Janiszewski† is the following: If $H$ and $K$ are two compact closed subsets of the plane neither of which separates the point $A$ from the point $B$ in the plane, and if $H \cdot K$ is connected, then $H+K$ does not separate $A$ from $B$ in the plane. With the aid of Theorem 13 this result can be generalized as follows.

Theorem 17. In a plane $S$ let $G=\left\{g_{i}\right\}$ be a countable collection of closed sets such that $G^{*}$ is compact. If $A$ and $B$ are two points of $S$, and $M$ and $N$ are two compact continua, each containing $A+B$, such that no element of $G$ intersects both $M$ and $N$, and such that the set of those points common to two or more elements of $G$ is contained in a complementary domain $D$ of $M+N$, then $G^{*}$ does not separate $A$ from $B$ in $S$.

For suppose the contrary. Then $(S-D) \cdot G^{*}$ separates $A$ from $B$ in $S-D$. Let $F$ be a closed subset of $(S-D) \cdot G^{*}$ which separates $A$ from $B$ in $S-D$. Since $S-D$ is a compactly connected continuum which does not separate $S$, and $F$ is compact, there exists, by Theorem 13, a component $F_{0}$ of $F$ which weakly disconnects $A$ from $B$ in $S-D$. Therefore $F_{0}$ contains points of both $M$ and $N$, and hence intersects at least two of the mutually exclusive closed sets $(S-D) \cdot g_{i}$. But this contradicts a theorem of Sierpinski.ł

THEOREM 18. Let $H$ and $K$ be two plane continua whose common part is not connected. If $N$ is a compact component of $H \cdot K$ such that $H \cdot K-N$ is closed, there exist two complementary domains $\Delta_{1}, \Delta_{2}$ of $H+K$ such that (1) the boundary of $\Delta_{i}(i=1,2)$ intersects $N, H-H \cdot K$, and $K-H \cdot K$, and (2) $\Delta_{1}+N$ $+\Delta_{2}$ contains a compact continuum $L$ such that $L \cdot \Delta_{1}$ and $L \cdot \Delta_{2}$ are non-vacuous connected sets.

Suppose first that $H$ and $K$ are compact.

Let $E$ be a compact closed set which separates $H-H \cdot K$ from $K-H \cdot K$ in $S$, the plane. Let $E^{\prime}$ denote the sum of $H \cdot K$ and those points of $E$ which lie in complementary domains of $H+K$ whose boundaries intersect both $H-H \cdot K$ and $K-H \cdot K$. The set $E^{\prime}$ is closed and separates $H-H \cdot K$ from $K-H \cdot K$ in $S$. Consider a component $h^{\prime}$ of $H-H \cdot K$ which has limit points in $N$ and in $H \cdot K-N$. Denote by $h$ the continuum which is the sum of $h^{\prime}$ and those components of $H \cdot K$ which contain limit points of $h^{\prime}$. Let $N$ be enclosed in a domain $D$ such that $\bar{D}$ contains no point of $H \cdot K-N$ and such

$\dagger$ Loc. cit.

$\ddagger$ Loc. cit. 
that the boundary $B$ of $D$ consists of a finite number of mutually exclusive simple closed curves. There exist two finite collections $T_{h}$ and $T_{K}$ of subarcs of $B$ such that (1) if $b$ and $c$ are elements of $T_{h}$ and $T_{K}$, respectively, then $b \cdot h \neq 0, c \cdot K \neq 0$, and $(b+c) \cdot E^{\prime}=b \cdot c=0$, and (2) $T_{h}{ }^{*} \supset h \cdot B$ and $T_{K}{ }^{*} \supset K \cdot B$. Denote by $H^{\prime}$ and $K^{\prime}$ the continua $h+T_{h}{ }^{*}$ and $K+T_{K}{ }^{*}$ respectively. We note that $H^{\prime} \cdot K^{\prime}=h \cdot K$. The set $E^{\prime}$ separates $H^{\prime}-H^{\prime} \cdot K^{\prime}$ from $K^{\prime}-H \cdot K$ in $S$. If $F$ represents the sum of $H \cdot K$ and those points of $E^{\prime}$ which lie in complementary domains of $H^{\prime}+K^{\prime}$ whose boundaries intersect both $H^{\prime}-H^{\prime} \cdot K^{\prime}$ and $K^{\prime}-H \cdot K$, then $F$ is a closed set which separates $H^{\prime}-H^{\prime} \cdot K^{\prime}$ from $K^{\prime}$ $-H \cdot K$ in $S$. The collection $R$ of those components of $\bar{D} \cdot\left(H^{\prime}+K^{\prime}\right)-N$ having no limit points in $N$ is finite. Let $J$ be a simple closed curve which lies in $D$, intersects no element of $R$, and separates $N$ from $H^{\prime} \cdot K^{\prime}-N$. There exists a subarc $P Q$ of $J$ whose end points lie in $H^{\prime}$ and $K^{\prime}$ respectively. The component $u$ of $\bar{D} \cdot\left(H^{\prime}+K^{\prime}\right)-N$ which contains $P$, and the component $v$ of $\bar{D} \cdot\left(H^{\prime}+K^{\prime}\right)-N$ which contains $Q$, have limit points in $N$. There exists a component $F_{1}$ of $F$ which separates $u$ from $v$ in $S$. Thus $F_{1}$ contains a point of $P Q$ and a point of $N$. Let $L_{1}$ be a subcontinuum of $F_{1}$ which is irreducible from $N$ to $J+B$, and denote by $d_{1}$ the complementary domain of $H^{\prime}+K^{\prime}$ which contains the connected set $L_{1}-L_{1} \cdot N$.

The continuum $S-d_{1}$ is compactly connected and does not separate the plane. Hence, by Theorem 13, there exists a subarc $P^{\prime} Q^{\prime}$ of $J$ which lies in $S-d_{1}$ and weakly disconnects $N$ from a point $X$ of $H^{\prime} \cdot K^{\prime}-N$ in $S-d_{1}$. There exists a subarc $p^{\prime} q^{\prime}$ of $P^{\prime} Q^{\prime}$ whose end points lie in $H^{\prime}$ and $K^{\prime}$ respectively. There exists, further, by Theorem 13 , a component $F_{2}$ of $F \cdot\left(S-d_{1}\right)$ which weakly disconnects $p^{\prime}$ from $q^{\prime}$ in $S-d_{1}$. Hence $F_{2}$ contains a point of $p^{\prime} q^{\prime}$. If $u^{\prime}$ and $v^{\prime}$ denote the components of $\bar{D} \cdot\left(H^{\prime}+K^{\prime}\right)-N$ which contain $p^{\prime}$ and $q^{\prime}$ respectively, then $u^{\prime}+N+v^{\prime}$ is a subcontinuum of $S-d_{1}$. Hence $F_{2}$ contains a point of $N$. Let $L_{2}$ be a subcontinuum of $F_{2}$ which is irreducible from $N$ to $J+B$, and denote by $d_{2}$ the complementary domain of $H^{\prime}+K^{\prime}$ which contains $L_{2}-L_{2} \cdot N$.

The complementary domains $\delta_{1}$ and $\delta_{2}$ of $h+K$ which contain $d_{1}$ and $d_{2}$, respectively, are distinct. For suppose the contrary. Let $Y_{1}$ and $Y_{2}$ be points of $d_{1}$ and $d_{2}$ respectively. There exists an $\operatorname{arc} \beta$ from $Y_{1}$ to $Y_{2}$ which lies in $\delta_{1}$ and hence contains no point of $h+K$. Since the boundaries of $d_{1}$ and $d_{2}$ intersect $K^{\prime}-H \cdot K$, there exist two arcs $Y_{1} Z_{1}$ and $Y_{2} Z_{2}$ which lie in $d_{1}$ and $d_{2}$, respectively, except for the points $Z_{1}$ and $Z_{2}$, which are contained in $K^{\prime}-H \cdot K$. The continuum $\Omega=Y_{1} Z_{1}+Y_{2} Z_{2}+K^{\prime}$ contains $Y_{1}+Y_{2}$ and has no point in common with $T_{h}{ }^{*}$. The common part of $T_{h}{ }^{*}$ and $h+K$ is a subset of $h^{\prime}$, and $h^{\prime}$ is a connected set having no point in common with $\beta+\Omega$. Hence, by Theorem $17, H^{\prime}+K=h+K+T_{h}{ }^{*}$ does not separate $Y_{1}$ from $Y_{2}$ in $S$. 
With this result it can be proved by a similar argument that $H^{\prime}+K^{\prime}$ does not separate $Y_{1}$ from $Y_{2}$ in $S$. But this is a contradiction.

It follows that the complementary domains $\Delta_{1}$ and $\Delta_{2}$ of $H+K$ which contain $L_{1}-L_{1} \cdot N$ and $L_{2}-L_{2} \cdot N$, respectively, are distinct. If we take $L=L_{1}$ $+N+L_{2}$, the domains $\Delta_{1}, \Delta_{2}$ are seen to satisfy the conditions of the theorem.

The case where $H$ and $K$ are not assumed to be compact can be reduced to the one considered by performing an inversion of the plane about a circle whose center lies in $S-(H+K)$.

COROLLARY. If $H$ and $K$ are two unbounded plane continua whose intersection is non-vacuous and compact, there exist two complementary domains $\Delta_{1}, \Delta_{2}$ of $H+K$ such that $(1) \Delta_{i}(i=1,2)$ contains an unbounded continuum, and (2) the boundary of $\Delta_{i}(i=1,2)$ intersects $H-H \cdot K$ and $K-H \cdot K$.

Theorem 19. A necessary and sufficient condition that a connected and locally arcwise connected subset $M$ of the plane be simply connected is that the interior of every simple closed curve lying in $M$ be a subset of $M$.

That the condition is sufficient follows from Theorem 7 and Lemma $\mathrm{K}$.

The condition is also necessary. For assume $M$ to be simply connected and suppose $M$ contains a simple closed curve $J$ whose interior $I$ contains a point $Q$ which does not lie in $M$. Let $l$ be a straight line which intersects $I$, and denote by $P_{1}$ and $P_{2}$ the two points of $l \cdot J$ such that the interval $P_{1} P_{2}$ of $l$ contains $l . J$. Join $P_{1}$ with $P_{2}$ by an $\operatorname{arc} b$ which contains $Q$ and lies in $I$ except for its end points. Let $A$ and $B$ denote interior points of the two arcs of $J$ whose end points are $P_{1}$ and $P_{2}$. The open curve $h=\left(l-P_{1} P_{2}\right)+b$ separates $A$ from $B$ in the plane. Hence $h \cdot M$ separates $A$ from $B$ in $M$. But no component of $h \cdot M$ can separate $A$ from $B$ in $M$; for such a component would contain $P_{1}+P_{2}$ and hence $Q$. This contradicts the hypothesis that $M$ is simply connected.

We get the following well known corollaries.

Corollary 1. A bounded, connected subdomain of the plane is simply connected if and only if its complement is connected. This remains true if "complement" is replaced by "boundary."

COROLlary 2. An unbounded, connected subdomain of the plane is simply connected if and only if every component of its complement is unbounded. This remains true if "complement" is replaced by "boundary."

COROLlaRy 3. Every complementary domain of a plane closed set each component of which is unbounded is simply connected. 
COROLlaRy 4. If $D$ is a complementary domain of a bounded plane continuum, and $F$ is a bounded and relatively closed subset of $D$ which separates a point $A$ from a point $B$ in $D$, then $F$ contains a connected subset which separates $A$ from $B$ in $D$.

An application of Corollary 3 and Theorem 2 is the following well known theorem: If $H$ and $K$ are two closed sets neither of which separates the point $A$ from the point $B$ in the plane, and if each component of $H \cdot K$ is unbounded, then $H+K$ does not separate $A$ from $B$ in the plane. This theorem can be generalized as follows:

THEOREM 20. In a connected and locally arcwise connected subset $M$ of a plane $S$ let $G$ be a countable collection of relatively closed sets such that (1) the common part of every pair of elements of $G$ is the set $H$ (which may be vacuous), (2) either $H+(S-M)$ is vacuous or every component of $H+(S-M)$ is unbounded, and (3) $G^{*}$ is locally compact in $M$. If no element of $G$ separates the point $A$ from the point $B$ in $M$, then $G^{*}$ does not separate $A$ from $B$ in $M$.

Let $b_{1}$ and $b_{2}$ be two arcs from $A$ to $B$ that lie in $M-H$. The set $H+(S$ $-M)$, if not vacuous, is contained in the unbounded domain $D$ which is complementary to $b_{1}+b_{2}$ in $S$. Hence the compact continuum $S-D$ is a subset of $M-H$. But $S-D$ does not separate $S$ and therefore, by Theorem $\mathrm{K}$, is simply connected in the weak sense. Hence, by Theorem $3, G^{*}$ does not separate $A$ from $B$ in $M$.

Another special case of this result is the following theorem of Anna Mullikin $\dagger$ : If $G$ is a countable collection of mutually exclusive closed sets lying in the plane $S$, and no element of $G$ separates the point $A$ from the point $B$ in $S$, then $G^{*}$ does not separate $A$ from $B$ in $S$.

If the collection $G$ is not restricted to be countable, we have a proposition related to a theorem $\ddagger$ of Rutt and Roberts:

THEOREM 21. In a connected and locally arcwise connected subset $M$ of a plane $S$ let $G$ be any collection of connected sets which are closed in $S$ such that (1) the common part of every pair of elements of $G$ is the non-vacuous set $H$, (2) every component of $H+(S-M)$ is unbounded, and (3) $G^{*}$ is closed in $M$. If no element of $G$ separates the point $A$ from the point $B$ in $M$, then $G^{*}$ does not separate $A$ from $B$ in $M$.

$\dagger$ Certain theorems relating to plane connected point sets, these Transactions, vol. 24 (1922), p. 148, Theorem 3.

$\ddagger$ See N. E. Rutt, On certain types of plane continua, these Transactions, vol. 33 (1931), p. 815, Theorem IV and Corollary IV; and J. H. Roberts, Concerning collections of continua not all bounded, American Journal of Mathematics, vol. 52 (1930), p. 553, Theorem I. 
In the outline of proof that follows let $S$ be the space of reference.

Suppose the theorem false. Select an element $g$ of $G$, choosing it to be unbounded if there are any unbounded elements of $G$. Let $L$ denote the collection of all elements each of which is the sum of $g$ and a component of a set obtained by subtracting $H$ from an element of $G$. There exists a subset $F$ of $L^{*}$ which is closed in $M$, separates $A$ from $B$ in $M$, contains every element of $L$ which has with it a point of $L^{*}-g$ in common, and is irreducible with respect to these three properties.

With the aid of Theorem 20 there can be constructed four $\operatorname{arcs} A P_{i}$ $(i=1, \cdots, 4)$ lying in $M$ such that (1) $A P_{i}(i=1, \cdots, 4)$ has in common with $F$ the point $P_{i}$ and this point only, and (2) $P_{1}, \cdots, P_{4}$ lie in $F-g$ and in distinct elements $l_{1}, \cdots, l_{4}$, respectively, of $L$. Let $J$ be a simple closed curve which separates $A$ from $F$ in $S$ such that, if $D$ denotes the complementary domain of $J$ that contains $F$, no two of the $\operatorname{arcs} A P_{i}$ have a point of $\bar{D}$ in common. Denote by $Q_{i}(i=1, \cdots, 4)$ the first point of $P_{i} A$ which lies on $J$. Two of the points $Q_{i}$, say $Q_{1}$ and $Q_{3}$, separate the other two in $J$. The set $W=Q_{1} P_{1}+Q_{3} P_{3}+l_{1}+l_{3}$ contains $Q_{1}+Q_{3}$ and lies in $\bar{D}$. Moreover $W$ is either a continuum or the sum of two or three unbounded continua. Therefore $W$ separates $Q_{2}$ from $Q_{4}$, and hence $P_{2}$ from $P_{4}$, in $\bar{D}$. It follows that $l_{1}+l_{3}$ separates $l_{2}-g$ from $l_{4}-g$ in $F$. Thus $F-\left(l_{1}+l_{3}\right)=F_{2}+F_{4}$, where $F_{2}$ and $F_{4}$ are mutually separated sets containing $l_{2}-g$ and $l_{4}-g$ respectively. The sets $R_{2}=F_{2}+\left(l_{1}+l_{3}\right)$ and $R_{4}=F_{4}+\left(l_{1}+l_{3}\right)$ are closed in $M$ and each contains every element of $L$ which has with it a point of $L^{*}-g$ in common. But $R_{2}$ and $R_{4}$ are proper subsets of $F$. Hence neither $R_{2}$ nor $R_{4}$ can separate $A$ from $B$ in $M$. Therefore, by Theorem $20, R_{2}+R_{4}(=F)$ does not separate $A$ from $B$ in $M$, contrary to construction.

THEOREM 22. If a bounded and locally arcwise connected subset $M$ of the plane $S$ separates the point $A$ from the point $B$ in $S$, then $M$ contains a simple closed curve which separates $A$ from $B$ in $S$.

By hypothesis $S-M=H+K$, where $H$ and $K$ are mutually separated sets containing $A$ and $B$ respectively. One of the sets $H, K$ is bounded since the exterior of a circle enclosing $M$ is a subset either of $H$ or of $K$. Hence there exists a compact continuum $F$ which separates $A$ from $B$ in $S$ and contains no point of $H+K$. By a theorem of R. L. Wilder $\dagger$ there exists a compact continuous curve $N$ which contains $F$ and is a subset of $M$; and by a theorem of R. L. Moore $\ddagger N$ contains a simple closed curve which separates $A$ from $B$ in $S$.

$\dagger$ Loc. cit.

$\ddagger$ Concerning continuous curves in the plane, Mathematische Zeitschrift, vol. 15 (1922), p. 260, Theorem 5 . 
This result enables us to generalize Corollary 1 of Theorem 19 as follows:

Theorem 23. A bounded, connected, and locally arcwise connected subset of the plane is simply connected if and only if it does not separate the plane.

As further applications of simply connected sets we shall prove several theorems relating to the separation of a continuum by a closed set.

THEOREM 24. Let $H$ and $K$ be two plane continua of which $K$ is compact if $H$ is compact. If $H$ disconnects the boundary of some complementary domain of $H+K$ then $H$ disconnects $K$.

Suppose the contrary. Let $B$ denote the boundary of a domain $\Delta$ complementary to $H+K$ such that $B-B \cdot H=N_{1}+N_{2}$, where $N_{1}$ and $N_{2}$ are mutually separated sets. The set $N_{1}+N_{2}$ is a subset of that complementary domain $D$ of $H$ which contains $\Delta$. Let $F$ be a relatively closed subset of $D$ which separates $N_{1}$ from $N_{2}$ in $D$, and let $F$ be compact if $H$ is compact. By Corollaries 3 and 4 of Theorem 19 there exists a component $F_{0}$ of $F$ that separates a point $P_{1}$ of $N_{1}$ from a point $P_{2}$ of $N_{2}$ in $D$. Hence $F_{0}$ contains a point of $\Delta$, which implies that $F_{0}$ is a subset of $\Delta$. But $K-K \cdot H$ is a connected subset of $D$ which contains $P_{1}$ and $P_{2}$ but no point of $\Delta$. This is a contradiction.

THEOREM 25. In an n-dimensional euclidean space $E_{n}$ the complement of every closed set $K$ of dimension $n-3$ or less is simply connected.

That the complement $D$ of $K$ is connected is well known. Suppose $D$ is not simply connected. Then, by Theorem $6, D=D_{1}+D_{2}$, where $D_{1}$ and $D_{2}$ are connected and relatively closed subsets of $D$ whose intersection is not connected. Thus $D_{1} \cdot D_{2}=I_{1}+I_{2}$, where $I_{1}$ and $I_{2}$ are mutually separated sets. Let $F$ be a closed set which separates $I_{1}$ from $I_{2}$ in $E_{n}$. Let $P_{1}$ and $P_{2}$ be points of $I_{1}$ and $I_{2}$ respectively. Then there exists a closed subset $B$ of $F$ which separates $P_{1}$ from $P_{2}$ in $E_{n}$ and is the common boundary of two domains. Since the dimension of $B \cdot K$ cannot exceed $n-3$, it follows from a theorem of P. Alexandroff $\dagger$ that $B-B \cdot K$ is a connected set. But $B-B \cdot K$ contains a point of $D_{1}$ and a point of $D_{2}$ and is a subset of $D_{1}+D_{2}$. Hence $B-B \cdot K$ contains a point of $D_{1} \cdot D_{2}$, contrary to the fact that $F$, which contains $B$, has no point in common with $D_{1} \cdot D_{2}$.

The theorem of Alexandroff referred to is, as he has pointed out, $\ddagger$ equivalent to the following theorem: If $M$ and $N$ are two closed subsets of $E_{n}$ neither of which separates the point $A$ from the point $B$ in $E_{n}$, and if the

$\dagger$ Sur les multiplicités cantoriennes et le théorème de Phragmèn-Brouwer généralisé, Comptes Rendus, vol. 183 (1926), pp. 722-724. In this paper the implicit assumption seems to be made that the common boundary is compact. That this restriction is not necessary follows from an argument by inversion.

$\ddagger$ Ibid. 
dimension of $M \cdot N$ does not exceed $n-3$, then $M+N$ does not separate $A$ from $B$ in $E_{n}$. We note that Theorem 25 is another formulation of the same result.

THEOREM 26. In $n$-dimensional euclidean space a closed set $K$ of dimension $n-3$ or less disconnects a continuum $M$ if and only if it disconnects the boundary of some complementary domain of $M$.

Clearly $K$ disconnects $M$ if and only if $K \cdot M$ (whose dimension does not exceed $n-3$ ) disconnects $M$.

Suppose $M-K \cdot M=M_{1}+M_{2}$, where $M_{1}$ and $M_{2}$ are mutually separated sets. Since $K \cdot M$ does not disconnect space there exists an arc $P_{1} P_{2}$ whose end points lie in $M_{1}$ and $M_{2}$, respectively, but which otherwise contains no point of $M$. The set $P_{1} P_{2}-\left(P_{1}+P_{2}\right)$ lies in a domain $\Delta$ complementary to $M$. If $B$ denotes the boundary of $\Delta$ we have $B-K \cdot B=B \cdot M_{1}+B \cdot M_{2}$, where $B \cdot M_{1}$ and $B \cdot M_{2}$ are mutually separated sets containing $P_{1}$ and $P_{2}$ respectively. Thus $K \cdot M$ disconnects the boundary of $\Delta$.

The sufficiency of the condition can be proved with the aid of Theorem 25 and an argument similar to that of Theorem 24 .

In particular a point of a continuum $M$ in three or more dimensions is a cut point of $M$ if and only if it is a cut point of the boundary of a complementary domain of $M$. It is interesting to note that this proposition, though true $\dagger$ for bounded continua in the plane, is not generally valid for unbounded plane continua.

As a second application of Theorem 3 we shall extend a result of R. L. Moore $\ddagger$ to $n$-dimensional euclidean space $(n>2)$.

THEOREM 27. In a euclidean space $E$ of three or more dimensions let $G$ be a countable collection of closed sets of which the common part of each pair is the point $O$. If no element of $G$ separates the point $A$ from the point $B$ in $E$, then $G^{*}$ does not separate $A$ from $B$ in $E$.

Let $b_{1}$ and $b_{2}$ be two arcs from $A$ to $B$ that lie in $E-O$. Consider two hyperspheres with centers at $O$ and such that $b_{1}+b_{2}$ lies in the domain $D$ included between them. $D$ is homeomorphic to $E-O$ and hence, by Theorem 25, is simply connected. It follows from Theorem 3 that $G^{*}$ does not separate $A$ from $B$ in $E$.

† See R. L. Moore, Concerning the common boundary of two domains, Fundamenta Mathematicae, vol. 6 (1924), p. 211, Theorem 8; and G. T. Whyburn, Concerning continua in the plane, these Transactions, vol. 29 (1927), p. 389, Theorem 19.

$\ddagger$ Foundations of Point Set Theory, p. 298, Theorem 113.

UnIVERSity of TEXas, Austin, Texas 